\title{
Lowering of the complexity of quantum chemistry methods by choice of representation
}

Narbe Mardirossian, James D. McClain, and Garnet Kin-Lic Chan

Citation: The Journal of Chemical Physics 148, 044106 (2018);

View online: https://doi.org/10.1063/1.5007779

View Table of Contents: http://aip.scitation.org/toc/jcp/148/4

Published by the American Institute of Physics

\section{Articles you may be interested in}

Editorial: JCP Communications-Updating a valued community resource

The Journal of Chemical Physics 148, 010401 (2018); 10.1063/1.5019731

On the difference between variational and unitary coupled cluster theories

The Journal of Chemical Physics 148, 044107 (2018); 10.1063/1.5011033

Communication: An improved linear scaling perturbative triples correction for the domain based local pairnatural orbital based singles and doubles coupled cluster method [DLPNO-CCSD(T)]

The Journal of Chemical Physics 148, 011101 (2018); 10.1063/1.5011798

Improving the accuracy of Møller-Plesset perturbation theory with neural networks

The Journal of Chemical Physics 147, 161725 (2017); 10.1063/1.4986081

Analytical energy gradients for explicitly correlated wave functions. I. Explicitly correlated second-order Møller-Plesset perturbation theory

The Journal of Chemical Physics 147, 214101 (2017); 10.1063/1.5003065

$\sigma$-SCF: A direct energy-targeting method to mean-field excited states

The Journal of Chemical Physics 147, 214104 (2017); 10.1063/1.5001262

\section{AIP | The Jounal of Chemical Physics}

PERSPECTIVES 


\title{
Lowering of the complexity of quantum chemistry methods by choice of representation
}

\author{
Narbe Mardirossian, ${ }^{\text {a) }}$ James D. McClain, and Garnet Kin-Lic Chan ${ }^{\text {b) }}$ \\ Division of Chemistry and Chemical Engineering, California Institute of Technology, Pasadena, \\ California 91125, USA
}

(Received 2 October 2017; accepted 2 January 2018; published online 23 January 2018)

\begin{abstract}
The complexity of the standard hierarchy of quantum chemistry methods is not invariant to the choice of representation. This work explores how the scaling of common quantum chemistry methods can be reduced using real-space, momentum-space, and time-dependent intermediate representations without introducing approximations. We find the scalings of exact Gaussian basis Hartree-Fock theory, secondorder Møller-Plesset perturbation theory, and coupled cluster theory (specifically, linearized coupled cluster doubles and the distinguishable cluster approximation with doubles) to be $\mathcal{O}\left(N^{3}\right), \mathcal{O}\left(N^{3}\right)$, and $\mathcal{O}\left(N^{5}\right)$, respectively, where $N$ denotes the system size. These scalings are not asymptotic and hold over all ranges of N. Published by AIP Publishing. https://doi.org/10.1063/1.5007779
\end{abstract}

\section{INTRODUCTION}

A great deal of progress in quantum chemistry comes from introducing approximations, for instance, to the structure of the wavefunction. For the conventional ladder of quantum chemistry methods (i.e., mean-field theory, perturbation theory, coupled cluster theory, etc.), such approximations lead to significant reductions in cost relative to the formal scaling of the methods. For example, within a Gaussian basis, the exact scaling of Hartree-Fock theory (HF), second-order Møller-Plesset perturbation theory (MP2), and coupled cluster theory with (singles and) doubles $(\mathrm{CC}(\mathrm{S}) \mathrm{D})$ is commonly accepted to be $\mathcal{O}\left(N^{4}\right), \mathcal{O}\left(N^{5}\right)$, and $\mathcal{O}\left(N^{6}\right)$, respectively, as a function of system size, $N$. However, by assuming locality in the wavefunction solutions, one can reduce the scaling of these methods to $\mathcal{O}(N) .{ }^{1-8}$ Similarly, tensor factorization (i.e., density fitting, Cholesky decomposition, orbital-specific corrections and pair natural orbitals, tensor hypercontraction, etc. $)^{9-16}$ and stochastic methods ${ }^{17-20}$ can yield reduced costs under different sets of assumptions and guarantees. For example, factorization methods exploit low-rank in either the solutions or the Hamiltonian, while stochastic methods exchange a deterministic guarantee of error for a probabilistic guarantee of variance.

In this short note, we will be concerned with an alternate strategy to reduce the cost of quantum chemistry methods. In particular, we will examine how we can change the complexity of a method simply by changing the underlying intermediate representations. While the choices of representations and approximations are commonly considered together, here we draw a distinction between the complexity lowering achieved through representation and that achieved through approximation. This is because changing the representation does not itself introduce assumptions into the structure of the solutions, and

a)Electronic mail: nmardirossian@berkeley.edu

b)Electronic mail: gkc1000@gmail.com in this sense, keeps the methods exact. To illustrate succinctly how representations yield a change in complexity while preserving exactness, consider the electronic Hamiltonian in three different bases: a general orbital basis, a plane-wave basis, and a real-space basis such as a grid,

$$
\begin{aligned}
H= & \sum_{i j} t_{i j} a_{i}^{\dagger} a_{j}+\sum_{i j k l} v_{i j k l} a_{i}^{\dagger} a_{j}^{\dagger} a_{k} a_{l}, \\
H= & \sum_{k_{1} k_{2}} t_{k_{1} k_{2}} a_{k_{1}}^{\dagger} a_{k_{2}}+\sum_{k_{1} k_{2} k_{3}} v_{k_{1} k_{2} k_{3} K} a_{k_{1}}^{\dagger} a_{k_{2}}^{\dagger} a_{k_{3}} a_{K} \\
& \left(K+k_{3}=k_{1}+k_{2}\right), \\
H= & \sum_{r r^{\prime}} t_{r r^{\prime}} a_{r}^{\dagger} a_{r^{\prime}}+\sum_{r r^{\prime}} V_{r r^{\prime}} n_{r} n_{r^{\prime}} .
\end{aligned}
$$

Each representation is exact in the sense that no systemspecific structure in the matrix elements is assumed, but the number of elements is $\mathcal{O}\left(N^{4}\right), \mathcal{O}\left(N^{3}\right)$, and $\mathcal{O}\left(N^{2}\right)$, respectively, without further approximations.

Using similar ideas, we will explore how a choice of representation affects the standard hierarchy of electronic structure methods. Assuming Coulombic interactions between particles, we find that the exact scaling of common Gaussian basis methods is $\mathcal{O}\left(N^{3}\right)$ for Hartree-Fock, $\mathcal{O}\left(N^{3}\right)$ for MP2, $\mathcal{O}\left(N^{5}\right)$ for linearized coupled cluster doubles ${ }^{21,22}$ (LCCD), and $\mathcal{O}\left(N^{5}\right)$ for the distinguishable cluster approximation with doubles ${ }^{23}$ (DCD). These scalings are not asymptotic but hold over any range of the system size, $N$. To reveal these scalings, we employ real-space, momentum-space, and time-dependent intermediate representations. None of these intermediate representations are new. Indeed, elements of our argument resemble those in the literature dating back to the earliest days of quantum chemistry. ${ }^{24}$ However, we will cleanly draw a line between the mathematical operations that retain exactness of the methods, and those that introduce assumptions into the solutions. In this way, the scalings we derive are clearly free from approximation. 


\section{HARTREE-FOCK THEORY}

As a warmup exercise to see how our results arise, consider the Hartree-Fock exchange energy. The conventional $\mathcal{O}\left(N^{4}\right)$ scaling of exact Hartree-Fock arises from the evaluation of all $\mathcal{O}\left(N^{4}\right)$ electron repulsion integrals, which are subsequently contracted into the one-particle density matrix, $\gamma$. However, at a more basic level, the Hartree-Fock exchange energy is simply a double integral,

$$
E_{\mathrm{HF}-\mathrm{X}}=-\iint \frac{\left|\gamma\left(\mathbf{r}_{1}, \mathbf{r}_{2}\right)\right|^{2}}{\left|\mathbf{r}_{1}-\mathbf{r}_{2}\right|} d \mathbf{r}_{1} d \mathbf{r}_{2}
$$

Given the integrand, this integral can be "exactly" evaluated by quadrature with $\mathcal{O}\left(N^{2}\right)$ cost, regardless of the form of $\gamma$. To be a little more precise, we use the term "exact" for quadrature because so long as singularities are appropriately handled, the cost to obtain a desired accuracy $\epsilon$ is polylogarithmic in $\epsilon$, i.e., $\sim \log (\epsilon)^{\alpha}$. To obtain the integrand, we must evaluate $\gamma$ (here expanded in a Gaussian basis) at the coordinates ( $\mathbf{r}_{\mathbf{1}}$, $\left.\mathbf{r}_{2}\right)$. From $\gamma\left(\mathbf{r}_{1}, \mathbf{r}_{2}\right)=\sum_{i} \phi_{i}^{*}\left(\mathbf{r}_{1}\right) \phi_{i}\left(\mathbf{r}_{2}\right)$, we see that this carries $\mathcal{O}\left(N^{3}\right)$ cost; thus the full cost of evaluating the exchange energy is $\mathcal{O}\left(N^{3}\right)$.

We can also consider the cost of obtaining the HartreeFock solution. Hartree-Fock theory is a variational theory, and we can use the cost of evaluating the Lagrangian derivative as a proxy for the cost of solving the equations. Since the Lagrangian is an algebraic function of the variational parameters in the density matrix, the rules of adjoint differentiation ${ }^{25}$ dictate that the cost of the derivative is also $\mathcal{O}\left(N^{3}\right)$. Thus solving the Hartree-Fock equations (for a fixed number of derivative steps) is also $\mathcal{O}\left(N^{3}\right)$ cost.

The $\mathcal{O}\left(N^{3}\right)$ scaling of Hartree-Fock is certainly not a new result: it was already well known from work on pseudo-spectral (PS) methods. ${ }^{26-28}$ The above exercise merely emphasizes that the $\mathcal{O}\left(N^{3}\right)$ scaling does not arise from any approximations but only from the intermediate representation. Thus it describes the complexity of the exact method.

\section{SECOND-ORDER MØLLER-PLESSET PERTURBATION THEORY}

Second-order Møller-Plesset perturbation theory (MP2) is perhaps the simplest route to including electron correlation effects. The conventional $\mathcal{O}\left(N^{5}\right)$ formal scaling arises from the atomic orbital to molecular orbital integral transformation necessary to evaluate the MP2 energy in a canonical basis. Our treatment of MP2 resembles that of HF in Sec. II in that we first express the MP2 energy as a multi-dimensional integral. We employ the time-dependent (i.e., Laplace transform ${ }^{29}$ ) representation of the MP2 energy, which rewrites the energy as an integral over space and imaginary time. The Laplace transform is a common component of reduced scaling MP2 methods especially in conjunction with local approximations, ${ }^{4}$ but here we emphasize that by itself it only corresponds to a choice of representation. We also employ a Fourier representation of the Coulomb operator to enable its fast application. All of these elements can be found in various earlier studies, such as in real-space versions of MP2 $2^{30,31}$ and from the tensor hypercontraction literature ${ }^{16,32}$ (related to the former via the connection to quadrature $\left.{ }^{33}\right)$. However, our emphasis here will be on the complexity of MP2 in an "exact" (i.e., polylogarithmic in error) formulation. The recent work by Schäfer $e t$ al. found that the MP2 algorithm could be exactly reformulated through a choice of representation to have only quartic scaling. ${ }^{34}$ Here we find that the scaling of exact MP2 can be further reduced to $\mathcal{O}\left(N^{3}\right)$. (After submission, we were informed of the work of Moussa $^{35}$ that presents a related analysis of the cost of exact MP2.)

As a single space-time integral, the two components of the MP2 energy, termed direct (MP2-J) and exchange (MP2-K), are

$$
\begin{aligned}
E_{\mathrm{MP2}-\mathrm{J}}= & 2 \int g_{o}\left(\mathbf{r}_{\mathbf{1}}, \mathbf{r}_{\mathbf{1}}^{\prime}, \tau\right) g_{o}\left(\mathbf{r}_{2}, \mathbf{r}_{\mathbf{2}}^{\prime}, \tau\right) g_{v}\left(\mathbf{r}_{\mathbf{1}}^{\prime}, \mathbf{r}_{\mathbf{1}}, \tau\right) \\
& \times g_{v}\left(\mathbf{r}_{\mathbf{2}}^{\prime}, \mathbf{r}_{\mathbf{2}}, \tau\right) v\left(\left|\mathbf{r}_{\mathbf{1}}-\mathbf{r}_{\mathbf{2}}\right|\right) v\left(\left|\mathbf{r}_{\mathbf{1}}^{\prime}-\mathbf{r}_{\mathbf{2}}^{\prime}\right|\right) d \mathbf{R} d \tau, \\
E_{\mathrm{MP} 2-\mathrm{K}}= & -\int g_{o}\left(\mathbf{r}_{\mathbf{1}}, \mathbf{r}_{\mathbf{2}}^{\prime}, \tau\right) g_{o}\left(\mathbf{r}_{2}, \mathbf{r}_{\mathbf{1}}^{\prime}, \tau\right) g_{v}\left(\mathbf{r}_{\mathbf{1}}^{\prime}, \mathbf{r}_{\mathbf{1}}, \tau\right) \\
& \times g_{v}\left(\mathbf{r}_{\mathbf{2}}^{\prime}, \mathbf{r}_{\mathbf{2}}, \tau\right) v\left(\left|\mathbf{r}_{\mathbf{1}}-\mathbf{r}_{\mathbf{2}}\right|\right) v\left(\left|\mathbf{r}_{\mathbf{1}}^{\prime}-\mathbf{r}_{\mathbf{2}}^{\prime}\right|\right) d \mathbf{R} d \tau,
\end{aligned}
$$

where $d \mathbf{R}$ denotes an integration over all spatial coordinates, $v\left(\left|\mathbf{r}-\mathbf{r}^{\prime}\right|\right)$ is the Coulomb operator, and $g_{o}\left(\mathbf{r}, \mathbf{r}^{\prime}, \tau\right)$ and $g_{v}(\mathbf{r}$, $\left.\mathbf{r}^{\prime}, \tau\right)$ are occupied and virtual Green's functions, respectively, defined as

$$
\begin{aligned}
& g_{o}\left(\mathbf{r}, \mathbf{r}^{\prime}, \tau\right)=\sum_{i} \phi_{i}^{*}(\mathbf{r}) \phi_{i}\left(\mathbf{r}^{\prime}\right) e^{-\epsilon_{i} \tau}, \\
& g_{v}\left(\mathbf{r}, \mathbf{r}^{\prime}, \tau\right)=\sum_{a} \phi_{a}^{*}(\mathbf{r}) \phi_{a}\left(\mathbf{r}^{\prime}\right) e^{\epsilon_{a} \tau} .
\end{aligned}
$$

To obtain the appropriate scaling of the algorithm, it is necessary to treat the convolution integrals with the Coulomb operator in special way. Within the Fourier representation, using a uniform mesh in real and momentum space, the well-known result is that the Coulomb potential, $J\left(\mathbf{r}^{\prime}\right)$, corresponding to a charge distribution, $\rho(\mathbf{r})$,

$$
J\left(\mathbf{r}^{\prime}\right)=\int \frac{\rho(\mathbf{r})}{\left|\mathbf{r}-\mathbf{r}^{\prime}\right|} d \mathbf{r}=(2 \pi)^{-3} \int \frac{4 \pi}{G^{2}} \rho(\mathbf{G}) e^{-i \mathbf{G} \cdot \mathbf{r}^{\prime}} d \mathbf{G},
$$

can be computed using the fast Fourier transform with $\mathcal{O}(N \log N)$ cost, which we will consider $\mathcal{O}(N)$ for simplicity. Errors due to periodic images can either be thought of as arising from the limits of integration in the quadrature or can be eliminated by truncating the Coulomb operator. ${ }^{36,37}$ Alternatively, one can compute the Coulomb potential on unstructured grids by solving the real-space Poisson equation with $\mathcal{O}(N)$ cost. ${ }^{38}$

In either case, assuming that the Coulomb operator can be applied at $\mathcal{O}(N)$ cost and assuming that Green's functions have been formed (which requires the same $\mathcal{O}\left(N^{3}\right)$ operation for both MP2 components), we can break down the evaluation of the MP2-J expression into the following steps: ${ }^{39,40}$

$$
\begin{aligned}
f\left(\mathbf{r}, \mathbf{r}^{\prime}, \tau\right) & =g_{o}\left(\mathbf{r}, \mathbf{r}^{\prime}, \tau\right) g_{v}\left(\mathbf{r}^{\prime}, \mathbf{r}, \tau\right), \\
F\left(\mathbf{r}^{\prime}, \mathbf{r}^{\prime \prime}, \tau\right) & =\int f\left(\mathbf{r}, \mathbf{r}^{\prime}, \tau\right) v\left(\left|\mathbf{r}-\mathbf{r}^{\prime \prime}\right|\right) d \mathbf{r}, \\
E_{\mathrm{MP} 2-\mathrm{J}} & =2 \int F\left(\mathbf{r}^{\prime}, \mathbf{r}^{\prime \prime}, \tau\right) F\left(\mathbf{r}^{\prime \prime}, \mathbf{r}^{\prime}, \tau\right) d \mathbf{R} d \tau .
\end{aligned}
$$


In the first step, the two pairs of occupied and virtual Green's functions that depend on the same real-space indices are combined at $\mathcal{O}\left(N^{2}\right)$ cost, while the second step scales as $\mathcal{O}\left(N^{2}\right)$ because it involves the application of the Coulomb operator at every point $\mathbf{r}^{\prime}$. Finally, the energy evaluation is a double integral and is thus of $\mathcal{O}\left(N^{2}\right)$ cost. Note that the latter result indicates that certain variants of MP2, such as scaled opposite-spin MP2, ${ }^{41}$ have an exact complexity of $\mathcal{O}\left(N^{2}\right)$ (aside from the formation of Green's functions).

The complexity of the MP2-K expression can be determined in a similar way. We group the expressions as follows:

$$
\begin{aligned}
G\left(\mathbf{r}_{1}^{\prime}, \mathbf{r}_{2}^{\prime}, \tau\right)= & \int d \mathbf{r}_{2}\left[\int d \mathbf { r } _ { 1 } \left(g_{o}\left(\mathbf{r}_{1}, \mathbf{r}_{\mathbf{2}}^{\prime}, \tau\right) g_{v}\left(\mathbf{r}_{\mathbf{1}}^{\prime}, \mathbf{r}_{1}, \tau\right)\right.\right. \\
& \left.\left.\times v\left(\left|\mathbf{r}_{\mathbf{1}}-\mathbf{r}_{2}\right|\right)\right) g_{o}\left(\mathbf{r}_{2}, \mathbf{r}_{1}^{\prime}, \tau\right) g_{v}\left(\mathbf{r}_{\mathbf{2}}^{\prime}, \mathbf{r}_{2}, \tau\right)\right], \\
E_{\mathrm{MP} 2-\mathrm{K}}= & -\int G\left(\mathbf{r}_{1}, \mathbf{r}_{2}, \tau\right) v\left(\left|\mathbf{r}_{1}-\mathbf{r}_{2}\right|\right) d \mathbf{R} d \tau .
\end{aligned}
$$

With MP2-K, the four Green's functions have unique pairs of indices and cannot be straightforwardly combined as in MP2$\mathrm{J}$. The first step above is the most expensive, as the convolution integral $(\mathcal{O}(N)$ cost $)$ is carried out for the $\mathcal{O}\left(N^{2}\right)$ pairs of grid points. Thus the entire MP2 energy can be determined at $\mathcal{O}\left(N^{3}\right)$ cost.

As a simple numerical demonstration of this algorithm, we have implemented an elementary cubic-scaling MP2 using the PySCF programming framework. ${ }^{42}$ We start from the integral expressions in Eqs. (5) and (6) and build the intermediates in Eqs. (7)-(14) on a uniform cubic grid. The Coulomb operator is applied using a three-dimensional fast Fourier transform. Instead of the scaling with system size, we here carry out the simpler test of scaling with respect to the number of cubic grid points, which for fixed accuracy is proportional to the system size. For the diamond cubic primitive cell (lattice constant of 6.74 Bohr, GTH-SZV basis set ${ }^{43}$ and GTH LDA pseudopotential $^{44}$ ), the timings and scalings are shown in Fig. 1 for a single Laplace point evaluation. The percent errors with respect to the large grid limit are given in Fig. 2. We see clearly that the

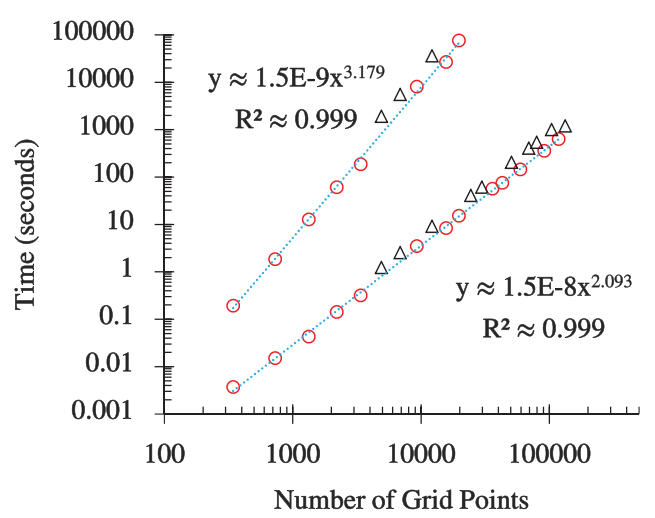

FIG. 1. Scaling for MP2-J (bottom) and MP2-K (top) within our pilot $\mathcal{O}\left(N^{3} \log N\right)$ MP2 implementation. The circles correspond to cubic grids with a length that is a factor of $2,3,5,7,11$, or 13 , while the triangles correspond to prime numbers or non-ideal lengths. The test system is a diamond cubic primitive cell at a lattice constant of $6.74 \mathrm{Bohr}$, using the GTH-SZV basis set and GTH LDA pseudopotential.

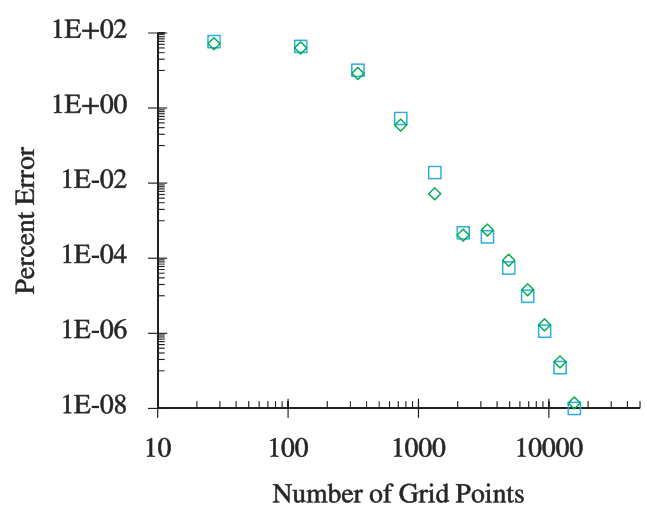

FIG. 2. Percent errors for MP2-J (blue squares) and MP2-K (green diamonds) within our pilot $\mathcal{O}\left(N^{3} \log N\right)$ MP2 implementation. The test system is a diamond cubic primitive cell at a lattice constant of $6.74 \mathrm{Bohr}$, using the GTH-SZV basis set and GTH LDA pseudopotential.

MP2-J algorithm scales close to quadratically with the number of grid points [its formal scaling in the implementation is $\mathcal{O}\left(N^{2} \log N\right)$ ], while the MP2-K algorithm scales close to cubically with the number of grid points. The cubic scaling and conventional evaluation of the Laplace transform MP2 energy agree to 12 significant figures.

\section{COUPLED CLUSTER THEORY}

The above general arguments can be repeated to derive lower formal complexities for a variety of different quantum chemistry methods. Here we will briefly outline how they can be extended to several coupled cluster approximations. Unlike in MP2, the coupled cluster amplitudes are not known explicitly but must be determined by solving the amplitude equations. For simplicity, we will discuss only the case of CCD (the singles contribution is subleading in complexity), where the $t_{2}$ amplitude is the four index tensor $t_{i j}^{a b}$. Conventionally, the cost of CCD is considered to be $\mathcal{O}\left(N^{6}\right)$. Here we show that certain subsets of diagrams that have $\mathcal{O}\left(N^{6}\right)$ cost (the LCCD and DCD subsets) can be reduced to $\mathcal{O}\left(N^{5}\right)$ cost without assuming any structure in the amplitudes. A similar asymptotic scaling in a plane-wave basis, using a tensor hypercontraction approximation for the integrals but also without assuming structure in the amplitudes, has recently been reported in Ref. 45 . Our analysis is related to that in Ref. 45 but illustrates that the $\mathcal{O}\left(N^{5}\right)$ scaling is an exact, rather than asymptotic, result.

The coupled cluster doubles correlation energy is given by the trace of the amplitudes with the integrals in Eq. (1) (assuming spin orbitals),

$$
\begin{aligned}
E_{\mathrm{CCD}} & =\frac{1}{4} \sum_{i j a b} t_{i j}^{a b} v_{i j a b} \\
& =\frac{1}{4} \int t\left(\mathbf{r}_{\mathbf{1}}, \mathbf{r}_{\mathbf{2}}, \mathbf{r}_{\mathbf{1}}, \mathbf{r}_{\mathbf{2}}\right) v\left(\left|\mathbf{r}_{\mathbf{1}}-\mathbf{r}_{\mathbf{2}}\right|\right) d \mathbf{r}_{\mathbf{1}} d \mathbf{r}_{\mathbf{2}},
\end{aligned}
$$

where the real-space amplitude is defined as

$$
\begin{aligned}
t\left(\mathbf{r}_{\mathbf{1}}, \mathbf{r}_{\mathbf{2}}, \mathbf{r}_{\mathbf{1}}^{\prime}, \mathbf{r}_{\mathbf{2}}^{\prime}\right)= & \sum_{i j a b} t_{i j}^{a b}\left[\phi_{i}^{*}\left(\mathbf{r}_{\mathbf{1}}\right) \phi_{j}^{*}\left(\mathbf{r}_{\mathbf{2}}\right) \phi_{a}\left(\mathbf{r}_{\mathbf{1}}^{\prime}\right) \phi_{b}\left(\mathbf{r}_{\mathbf{2}}^{\prime}\right)\right. \\
& \left.-\phi_{i}^{*}\left(\mathbf{r}_{\mathbf{1}}\right) \phi_{j}^{*}\left(\mathbf{r}_{\mathbf{2}}\right) \phi_{b}\left(\mathbf{r}_{\mathbf{1}}^{\prime}\right) \phi_{a}\left(\mathbf{r}_{\mathbf{2}}^{\prime}\right)\right] .
\end{aligned}
$$


The coupled cluster energy is a double integral and thus given the real-space amplitudes, requires $\mathcal{O}\left(N^{2}\right)$ cost. However, the amplitude equations do not define the amplitudes in this form, and the transformation from the orbital basis to real-space is of $\mathcal{O}\left(N^{5}\right)$ cost. Thus the exact cost to evaluate the coupled cluster energy, assuming the use of the orbital-based amplitude equations to determine the amplitudes, is $\mathcal{O}\left(N^{5}\right)$, without further approximations. (If the amplitudes can be defined in a different way, for example, as in the random-phase approximation to CCD, this cost can be reduced.)

The CCD amplitude equations ${ }^{46}$ are conveniently presented in a diagrammatic form in Fig. 3. Above each diagram, we give the scaling of each term. Like the above argument for the energy, we can transform the indices of each amplitude into the real-space representation as needed to apply the Coulomb operator, before transforming back into the orbital basis. This change of representation reduces the complexity of nine of the 20 terms from $\mathcal{O}\left(N^{6}\right)$ to $\mathcal{O}\left(N^{5}\right)$. These reductions are also indicated in Fig. 3. According to the diagrams, LCCD corresponds to the first nine terms, while DCD corresponds to LCCD, plus the three other terms whose scaling is reduced from $\mathcal{O}\left(N^{6}\right)$ to $\mathcal{O}\left(N^{5}\right)\left(\mathrm{D}_{3 b}, \mathrm{D}_{3 b x 1}\right.$, and $\left.\mathrm{D}_{3 b \times 2}\right)$, plus the last four $\mathcal{O}\left(N^{5}\right)$ terms. Thus the exact cost to determine the amplitudes in either LCCD or DCD is $\mathcal{O}\left(N^{5}\right)$.

$$
\begin{gathered}
\frac{1}{2} \sum_{C D}\langle A B|\hat{v}| C D\rangle t_{I J}^{C D} \\
\frac{1}{2} \sum_{C D} \int A\left(\mathbf{r}_{1}\right) B\left(\mathbf{r}_{2}\right) v\left(\mathbf{r}_{1}, \mathbf{r}_{2}\right) C\left(\mathbf{r}_{1}\right) D\left(\mathbf{r}_{2}\right) t_{I J}^{C D} d \mathbf{r}_{1} d \mathbf{r}_{2} \\
t_{I J}^{D}\left(\mathbf{r}_{1}\right)=\sum_{C} C\left(\mathbf{r}_{1}\right) t_{I J}^{C D} \quad t_{I J}\left(\mathbf{r}_{1}, \mathbf{r}_{2}\right)=\sum_{D} D\left(\mathbf{r}_{2}\right) t_{I J}{ }^{D}\left(\mathbf{r}_{1}\right) \\
T_{I J}\left(\mathbf{r}_{1}, \mathbf{r}_{2}\right)=t_{I J}\left(\mathbf{r}_{1}, \mathbf{r}_{2}\right) v\left(\mathbf{r}_{1}, \mathbf{r}_{2}\right) \\
T_{I J}^{A}\left(\mathbf{r}_{2}\right)=\int A\left(\mathbf{r}_{1}\right) T_{I J}\left(\mathbf{r}_{1}, \mathbf{r}_{2}\right) d \mathbf{r}_{1} \\
T_{I J}^{A B}=\frac{1}{2} \int B\left(\mathbf{r}_{2}\right) T_{I J}^{A}\left(\mathbf{r}_{2}\right) d \mathbf{r}_{2} .
\end{gathered}
$$

The nine diagrams with reduced complexity can be grouped into three separate types: (1) $\mathrm{D}_{2 c}, \mathrm{D}_{2 d}, \mathrm{D}_{2 e x 2}$, and $\mathrm{D}_{2 e x 3}$, (2) $\mathrm{D}_{2 e}$ and $\mathrm{D}_{2 e x 1}$, and (3) $\mathrm{D}_{3 b}, \mathrm{D}_{3 b x 1}$, and $\mathrm{D}_{3 b x 2}$. The type 1 terms contain a single $t_{2}$ amplitude, with the contraction indices corresponding to different electron coordinates (i.e., $\mathbf{r}_{1}$ and $\mathbf{r}_{2}$ ), while the type 2 terms contain a single $t_{2}$ amplitude, with the contraction indices corresponding to the same electron coordinate (i.e., either both $\mathbf{r}_{1}$ or both $\mathbf{r}_{2}$ ). The type 3 terms, despite containing a pair of $t_{2}$ amplitudes, can be evaluated in $\mathcal{O}\left(N^{5}\right)$ time because the contraction indices contained in each amplitude correspond to the same electron coordinate. To illustrate the scaling reduction for the three aforementioned types, we take a single term from each case and define appropriate intermediates in Eqs. (17)-(19), where Eq. (17) corresponds to diagram $\mathrm{D}_{2 c}$, Eq. (18) corresponds to diagram $\mathrm{D}_{2 e}$, and Eq. (19) corresponds to diagram $\mathrm{D}_{3 b}$,

$$
\begin{gathered}
2 \sum_{K C}\langle K B|\hat{v}| C J\rangle t_{I K}^{A C} \\
2 \sum_{K C} \int K\left(\mathbf{r}_{\mathbf{1}}\right) B\left(\mathbf{r}_{\mathbf{2}}\right) v\left(\mathbf{r}_{\mathbf{1}}, \mathbf{r}_{\mathbf{2}}\right) C\left(\mathbf{r}_{\mathbf{1}}\right) J\left(\mathbf{r}_{\mathbf{2}}\right) t_{I K}^{A C} d \mathbf{r}_{\mathbf{1}} d \mathbf{r}_{\mathbf{2}} \\
t_{I}^{A C}\left(\mathbf{r}_{\mathbf{1}}\right)=\sum_{K} K\left(\mathbf{r}_{\mathbf{1}}\right) t_{I K}^{A C} \quad t_{I}^{A}\left(\mathbf{r}_{\mathbf{1}}\right)=\sum_{C} C\left(\mathbf{r}_{1}\right) t_{I}^{A C}\left(\mathbf{r}_{\mathbf{1}}\right) \\
T_{I}^{A}\left(\mathbf{r}_{2}\right)=\int t_{I}^{A}\left(\mathbf{r}_{\mathbf{1}}\right) v\left(\mathbf{r}_{\mathbf{1}}, \mathbf{r}_{2}\right) d \mathbf{r}_{\mathbf{1}} \\
T_{I J}^{A B}=2 \int B\left(\mathbf{r}_{2}\right) J\left(\mathbf{r}_{2}\right) T_{I}^{A}\left(\mathbf{r}_{2}\right) d \mathbf{r}_{2} .
\end{gathered}
$$

In order to clarify the reduction in scaling, we will walk through the derivation for the $\mathrm{D}_{2 c}$ term. In a Gaussian basis, it is evident that this term scales as $\mathcal{O}\left(N^{6}\right)$. After rewriting the integral in its real-space form, the contractions over $\mathrm{C}$ and $\mathrm{D}$ each require $\mathcal{O}\left(N^{5}\right)$ time since the former involves four orbital indices and one real-space index and the latter involves three orbital indices and two real-space indices. Then, the result is multiplied by the Coulomb operator in real space, at $\mathcal{O}\left(N^{4}\right)$ cost. The next step is similar to that shown in Eq. (9) and scales as $\mathcal{O}\left(N^{4}\right)$, while the final step is again $\mathcal{O}\left(N^{5}\right)$. Thus, the scaling for a term that is conventionally $\mathcal{O}\left(N^{6}\right)$ can be exactly reduced
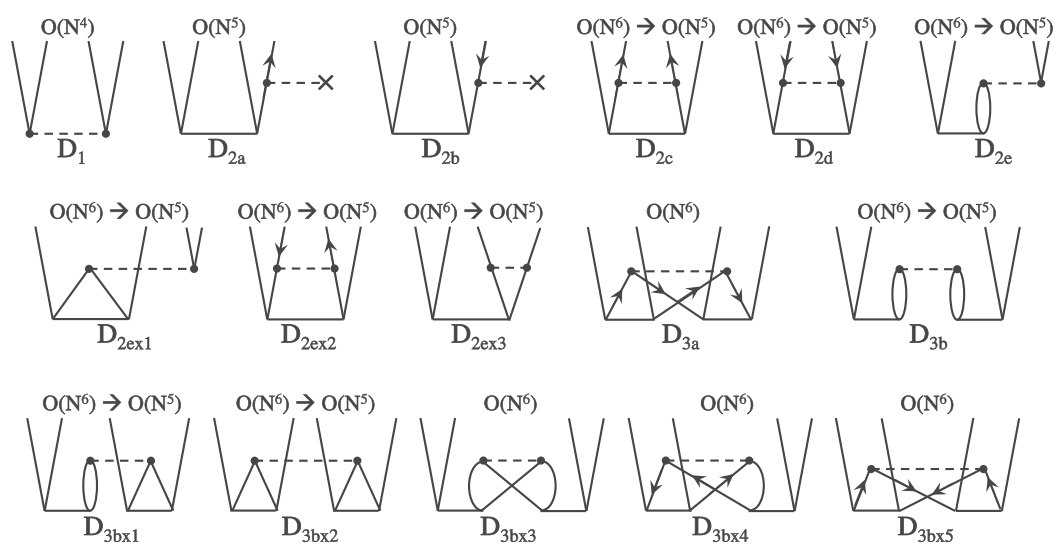

FIG. 3. Spin-summed CCD amplitude equation diagrams. The cost of diagrams associated with a single scaling is unchanged when using a real-space intermediate representation, while those with arrows experience scaling reduction [i.e., the scaling of term $\mathrm{D}_{2 c}$ is reduced from $\mathcal{O}\left(N^{6}\right)$ to $\mathcal{O}\left(N^{5}\right)$ ].

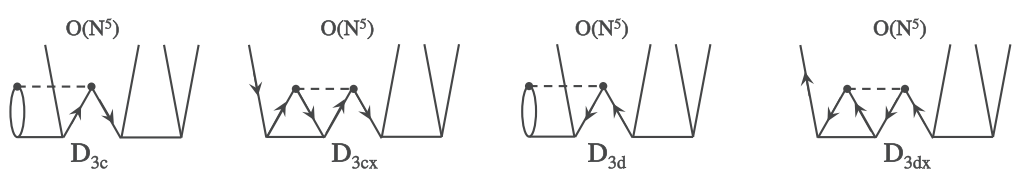


to $\mathcal{O}\left(N^{5}\right)$.

$$
\begin{gathered}
2 \sum_{K L C D}\langle K L|\hat{v}| C D\rangle t_{I K}^{A C} t_{L J}^{D B} \\
2 \sum_{K L C D} \int K\left(\mathbf{r}_{1}\right) L\left(\mathbf{r}_{2}\right) v\left(\mathbf{r}_{1}, \mathbf{r}_{2}\right) C\left(\mathbf{r}_{1}\right) D\left(\mathbf{r}_{2}\right) t_{I K}^{A C} t_{L J}^{D B} d \mathbf{r}_{\mathbf{1}} d \mathbf{r}_{\mathbf{2}} \\
t_{I}^{A C}\left(\mathbf{r}_{\mathbf{1}}\right)=\sum_{K} K\left(\mathbf{r}_{\mathbf{1}}\right) t_{I K}^{A C} \quad t_{I}^{A}\left(\mathbf{r}_{\mathbf{1}}\right)=\sum_{C} C\left(\mathbf{r}_{\mathbf{1}}\right) t_{I}^{A C}\left(\mathbf{r}_{\mathbf{1}}\right) \\
t_{J}^{D B}\left(\mathbf{r}_{\mathbf{2}}\right)=\sum_{L} L\left(\mathbf{r}_{2}\right) t_{L J}^{D B} \quad t_{J}^{B}\left(\mathbf{r}_{2}\right)=\sum_{D} D\left(\mathbf{r}_{\mathbf{2}}\right) t_{J}^{D B}\left(\mathbf{r}_{2}\right) \\
T_{I}^{A}\left(\mathbf{r}_{2}\right)=\int t_{I}^{A}\left(\mathbf{r}_{1}\right) v\left(\mathbf{r}_{1}, \mathbf{r}_{2}\right) d \mathbf{r}_{1} \\
T_{I J}^{A B}=2 \int t_{J}^{B}\left(\mathbf{r}_{2}\right) T_{I}^{A}\left(\mathbf{r}_{2}\right) d \mathbf{r}_{2} .
\end{gathered}
$$

\section{ALTERNATE REPRESENTATIONS}

In our above arguments, we reduced the exact scalings of quantum chemistry methods by combining several different representations. In all three methods (HF, MP2, and $\mathrm{CC}$ ), we used a real-space intermediate representation. We obtained additional cost reductions from the Fourier representation of the Coulomb operator, while the MP2 algorithm also used a time-dependent representation. These intermediate representations are not the only ones that lead to reduced scalings, and other choices may lead to lower computational prefactors. For example, if we allow for a polylogarithmic dependence of computational cost on the threshold error $\epsilon$, then we can regard atomic orbital bases as a form of exponentially localized real-space basis. This is the standard argument for atomic orbital screening, but here we are interested only in the reduction in complexity that can be achieved without assuming locality in the wavefunction or by cutting off algebraically decaying quantities (such as $R^{-6}$ contributions to the correlation energy ${ }^{47,48}$ ) that destroy the polylogarithmic computational cost. Within this sense of retaining the exactness of the method, as long as we also use a scheme to apply the Coulomb operator with $\mathcal{O}(N)$ cost, one recovers the same complexities we have derived above for systems on length scales larger than the atomic orbital size.

One way to apply the Coulomb operator in a fast scheme is to use a mixed basis and grid representation, as is commonly done in mixed Gaussian and plane-wave implementations ${ }^{36,49-51}$ where the Coulomb operator is applied, as above, in the Fourier representation. As an explicit example, we outline how to evaluate the MP2-J term using this idea as well as an atomic orbital representation. Here we use the standard Roman and Greek symbols for molecular orbitals and atomic orbitals, respectively, with the molecular orbitals expanded as $\phi_{p}(\mathbf{r})=\sum_{\mu} C_{\mu p} \mu(\mathbf{r})$. Contributions of atomic orbital products $\mu(\mathbf{r}) v(\mathbf{r})$ will be assumed screened if $\|\mu \nu\|<\epsilon$, and screened pairs will be indicated by the symbol $\langle\mu v\rangle$. Starting with the atomic orbital Laplace transform expression for MP2-J,

$$
\begin{aligned}
E_{\mathrm{MP2}-\mathrm{J}}= & 2 \int \sum_{\mu^{\prime} \nu^{\prime} \sigma^{\prime} \lambda^{\prime}} \sum_{\mu \nu \lambda \lambda}\left(\mu^{\prime} v^{\prime} \mid \sigma^{\prime} \lambda^{\prime}\right)(v \mu \mid \lambda \sigma) \\
& \times g_{\mu^{\prime} \mu} g_{\sigma^{\prime} \sigma} \bar{g}_{\nu^{\prime} \nu} \bar{g}_{\lambda^{\prime} \lambda} d \tau
\end{aligned}
$$

with the atomic orbital Green's functions [that require $\mathcal{O}\left(N^{3}\right)$ time to compute] defined as

$$
\begin{aligned}
& g_{\mu^{\prime} \mu}(\tau)=\sum_{i} C_{\mu^{\prime} i} C_{\mu i} e^{-\epsilon_{i} \tau}, \\
& \bar{g}_{\nu^{\prime} v}(\tau)=\sum_{a} C_{\nu^{\prime} a} C_{v a} e^{\epsilon_{a} \tau},
\end{aligned}
$$

it is possible to formulate a series of steps to evaluate Eq. (20) where the cost is no greater than $\mathcal{O}\left(N^{3}\right)$. The first three intermediates require $\mathcal{O}\left(N^{3}\right)$ cost,

$$
\begin{aligned}
\rho_{\mu}(\mathbf{r}, \tau) & =\sum_{\mu^{\prime}} \mu^{\prime}(\mathbf{r}) g_{\mu^{\prime} \mu}(\tau), \\
h_{\langle\mu \nu\rangle}(\mathbf{r}, \tau) & =\int \rho_{\mu}\left(\mathbf{r}^{\prime}, \tau\right) \rho_{\nu}\left(\mathbf{r}^{\prime}, \tau\right) v\left(\left|\mathbf{r}-\mathbf{r}^{\prime}\right|\right) d \mathbf{r}^{\prime}, \\
h_{\langle\mu \nu\rangle\left\langle\sigma^{\prime} \lambda^{\prime}\right\rangle}(\tau) & =\int h_{\langle\mu \nu\rangle}(\mathbf{r}, \tau)\left\langle\sigma^{\prime} \lambda^{\prime}\right\rangle(\mathbf{r}) d \mathbf{r},
\end{aligned}
$$

and the final energy evaluation,

$$
E_{\mathrm{MP2}-\mathrm{J}}=2 \int \sum_{\left\langle\sigma^{\prime} \lambda^{\prime}\right\rangle,\langle\mu \nu\rangle} h_{\langle\mu \nu\rangle\left\langle\sigma^{\prime} \lambda^{\prime}\right\rangle}(\tau) h_{\left\langle\lambda^{\prime} \sigma^{\prime}\right\rangle\langle\nu \mu\rangle}(\tau) d \tau,
$$

is $\mathcal{O}\left(N^{2}\right)$ cost. Note that there are additional cubic steps in the evaluation of the MP2-J term compared to the quadrature implementation because in the case of quadrature, the cubic cost is confined to the formation of Green's functions at the beginning of the algorithm, while here the cubic cost is delayed until quantities are placed on the grid. However, it is clear that by using a mixture of atomic orbitals and quadrature, the overall prefactor is greatly reduced, as typically the number of atomic orbitals required in the "function" quadrature is much less than the number of grid points required for numerical quadrature. Although the above algorithm will exhibit cubic scaling on length scales determined only by the atomic orbitals rather than the locality of the wavefunction, the use of diffuse functions will prevent the onset of this scaling until larger systems. In such a case, alternative representations may prove useful, and this is a topic of future work.

\section{CONCLUSION}

In summary, the present work re-examines the exact scaling of several traditional quantum chemistry methods. We find that the freedom of choice of intermediates means that HF and MP2 can be reduced to cubic scaling, and variants of coupled cluster such as linearized coupled cluster doubles (LCCD) and the distinguishable cluster approximation with doubles (DCD) may be reduced to $\mathcal{O}\left(N^{5}\right)$ scaling. These are scalings of the exact methods in the sense that no assumptions are made about the forms of the solutions. The exact scalings that we describe encourage a modified perspective on several topics. For example, they lead to a different organization of the correlation hierarchy, where the complexity gap between density functional methods $\mathrm{s}^{52}$ and traditional wavefunction methods is eliminated. They also suggest a new way to classify diagrams in coupled cluster theory ${ }^{53}$ that may lead to new correlation approximations. Finally, given that the exact scalings are lower than that of many current approximate methods, substantial further 
reductions in cost can be obtained in practice by combining the ideas here with the rich existing set of techniques used to define approximate quantum chemistry methods.

\section{ACKNOWLEDGMENTS}

This work was supported by the US National Science Foundation through NSF: Nos. CHE 1665333 and NSF:SSI 1657286. G.K.-L.C. is a Simons Investigator in Theoretical Physics.

${ }^{1}$ E. Schwegler and M. Challacombe, J. Chem. Phys. 105, 2726 (1996).

${ }^{2}$ M. Challacombe and E. Schwegler, J. Chem. Phys. 106, 5526 (1997).

${ }^{3}$ C. Ochsenfeld, C. A. White, and M. Head-Gordon, J. Chem. Phys. 109, 1663 (1998).

${ }^{4}$ M. Schütz, G. Hetzer, and H.-J. Werner, J. Chem. Phys. 111, 5691 (1999).

${ }^{5}$ G. E. Scuseria and P. Y. Ayala, J. Chem. Phys. 111, 8330 (1999).

${ }^{6}$ M. Schütz and H.-J. Werner, J. Chem. Phys. 114, 661 (2001).

${ }^{7}$ S. Saeb $\varnothing$ and P. Pulay, J. Chem. Phys. 115, 3975 (2001).

${ }^{8}$ P. Pinski, C. Riplinger, E. F. Valeev, and F. Neese, J. Chem. Phys. 143, 034108 (2015).

${ }^{9}$ W. Meyer, J. Chem. Phys. 58, 1017 (1973).

${ }^{10}$ M. Sierka, A. Hogekamp, and R. Ahlrichs, J. Chem. Phys. 118, 9136 (2003).

${ }^{11}$ H.-J. Werner, F. R. Manby, and P. J. Knowles, J. Chem. Phys. 118, 8149 (2003).

${ }^{12}$ H. Koch, A. S. de Merás, and T. B. Pedersen, J. Chem. Phys. 118, 9481 (2003).

${ }^{13}$ F. Aquilante, T. B. Pedersen, and R. Lindh, J. Chem. Phys. 126, 194106 (2007).

${ }^{14}$ F. Neese, F. Wennmohs, and A. Hansen, J. Chem. Phys. 130, 114108 (2009).

${ }^{15}$ J. Yang, Y. Kurashige, F. R. Manby, and G. K. L. Chan, J. Chem. Phys. 134, 044123 (2011).

${ }^{16}$ E. G. Hohenstein, R. M. Parrish, and T. J. Martínez, J. Chem. Phys. 137, 044103 (2012)

${ }^{17}$ A. J. W. Thom and A. Alavi, Phys. Rev. Lett. 99, 143001 (2007).

${ }^{18}$ S. Y. Willow, K. S. Kim, and S. Hirata, J. Chem. Phys. 137, 204122 (2012).

${ }^{19}$ D. Neuhauser, E. Rabani, and R. Baer, J. Chem. Theory Comput. 9, 24 (2013).

${ }^{20}$ Q. Ge, Y. Gao, R. Baer, E. Rabani, and D. Neuhauser, J. Phys. Chem. Lett. 5, 185 (2014).

${ }^{21}$ R. J. Bartlett, Annu. Rev. Phys. Chem. 32, 359 (1981).

${ }^{22}$ A. G. Taube and R. J. Bartlett, J. Chem. Phys. 130, 144112 (2009).

${ }^{23}$ D. Kats and F. R. Manby, J. Chem. Phys. 139, 021102 (2013).

${ }^{24}$ S. Boys and P. Rajagopal, Quantum Calculations: Which Are Accumulative in Accuracy, Unrestricted in Expansion Functions, and Economical in
Computation, Advances in Quantum Chemistry series, Vol. 2 (Academic Press, 1966), pp. 1-24.

${ }^{25}$ L. B. Rall, Automatic Differentiation: Techniques and Applications (Springer, 1981).

${ }^{26}$ R. A. Friesner, Chem. Phys. Lett. 116, 39 (1985).

${ }^{27}$ M. N. Ringnalda, M. Belhadj, and R. A. Friesner, J. Chem. Phys. 93, 3397 (1990).

${ }^{28}$ R. B. Murphy, M. D. Beachy, R. A. Friesner, and M. N. Ringnalda, J. Chem. Phys. 103, 1481 (1995).

${ }^{29}$ M. Häser and J. Almlöf, J. Chem. Phys. 96, 489 (1992).

${ }^{30}$ F. A. Bischoff, R. J. Harrison, and E. F. Valeev, J. Chem. Phys. 137, 104103 (2012).

${ }^{31}$ S. Hirata, T. Shiozaki, C. M. Johnson, and J. D. Talman, Mol. Phys. 115, 510 (2017).

${ }^{32}$ C. Song and T. J. Martínez, J. Chem. Phys. 144, 174111 (2016).

${ }^{33}$ R. M. Parrish, E. G. Hohenstein, N. F. Schunck, C. D. Sherrill, and T. J. Martínez, Phys. Rev. Lett. 111, 132505 (2013).

${ }^{34}$ T. Schäfer, B. Ramberger, and G. Kresse, J. Chem. Phys. 146, 104101 (2017).

${ }^{35}$ J. E. Moussa, J. Chem. Phys. 140, 014107 (2014).

${ }^{36}$ L. Füsti-Molnar and P. Pulay, J. Chem. Phys. 116, 7795 (2002).

${ }^{37}$ L. Füsti-Molnár and P. Pulay, J. Mol. Struct.: THEOCHEM 666, 25 (2003).

${ }^{38}$ S. R. White, J. W. Wilkins, and M. P. Teter, Phys. Rev. B 39, 5819 (1989).

${ }^{39}$ H. N. Rojas, R. W. Godby, and R. J. Needs, Phys. Rev. Lett. 74, 1827 (1995).

${ }^{40}$ M. Kaltak, J. Klimeš, and G. Kresse, Phys. Rev. B 90, 054115 (2014).

${ }^{41}$ Y. Jung, R. C. Lochan, A. D. Dutoi, and M. Head-Gordon, J. Chem. Phys. 121, 9793 (2004).

${ }^{42}$ Q. Sun, T. C. Berkelbach, N. S. Blunt, G. H. Booth, S. Guo, Z. Li, J. Liu, J. D. McClain, E. R. Sayfutyarova, S. Sharma, S. Wouters, and G. K.-L. Chan, Wiley Interdiscip. Rev.: Comput. Mol. Sci. 8, e1340 (2018).

${ }^{43}$ J. VandeVondele and J. Hutter, J. Chem. Phys. 127, 114105 (2007).

${ }^{44}$ C. Hartwigsen, S. Goedecker, and J. Hutter, Phys. Rev. B 58, 3641 (1998).

${ }^{45}$ F. Hummel, T. Tsatsoulis, and A. Grüneis, J. Chem. Phys. 146, 124105 (2017).

${ }^{46}$ I. Shavitt and R. J. Bartlett, Many-Body Methods in Chemistry and Physics: MBPT and Coupled-Cluster Theory (Cambridge University Press, 2009).

${ }^{47}$ A. K. Wilson and J. Almlöf, Theor. Chim. Acta 95, 49 (1997).

${ }^{48}$ B. Doser, D. S. Lambrecht, J. Kussmann, and C. Ochsenfeld, J. Chem. Phys. 130, 064107 (2009).

${ }^{49}$ J. Hutter, M. Iannuzzi, F. Schiffmann, and J. VandeVondele, Wiley Interdiscip. Rev.: Comput. Mol. Sci. 4, 15 (2014).

${ }^{50}$ J. McClain, Q. Sun, G. K.-L. Chan, and T. C. Berkelbach, J. Chem. Theory Comput. 13, 1209 (2017).

${ }^{51}$ Q. Sun, T. C. Berkelbach, J. D. McClain, and G. K. Chan, J. Chem. Phys. 147, 164119 (2017).

${ }^{52}$ N. Mardirossian and M. Head-Gordon, Mol. Phys. 115, 2315 (2017).

${ }^{53}$ R. J. Bartlett and M. Musiał, Rev. Mod. Phys. 79, 291 (2007). 